\title{
Protocol
}

\section{Impact and risk factors for clinically relevant surgery-related muscle loss in patients after major abdominal cancer surgery: study protocol for a prospective observational cohort study (MUSCLE POWER)}

\author{
Judith E. K. R. Hentzen ${ }^{1}$, Laura van Wijk ${ }^{1}$, Carlijn I. Buis ${ }^{1}$, Alain R. Viddeleer ${ }^{2}$, \\ Geertruida H. de Bock ${ }^{3}$, Cees P. van der Schans ${ }^{4,5}$, Gooitzen M. van Dam ${ }^{1,6}$, \\ Schelto Kruijff ${ }^{1}$, Joost M. Klaase ${ }^{1}$
}

\begin{abstract}
${ }^{1}$ Department of Surgery, ${ }^{2}$ Department of Radiology, ${ }^{3}$ Department of Epidemiology, ${ }^{4}$ Department of Rehabilitation and Health Psychology, ${ }^{6}$ Department of Nuclear Medicine and Molecular Imaging and Intensive Care, University of Groningen, University Medical Center Groningen, Groningen, The Netherlands

${ }^{5}$ Research Group Healthy Ageing, Allied Health Care and Nursing, Center of Expertise Healthy Ageing, Hanze University of Applied Sciences, Groningen, The Netherlands
\end{abstract}

Received: 19 April 2019

Revised: 28 May 2019

Accepted: 05 June 2019

\section{*Correspondence:}

Dr. J.E.K.R. Hentzen,

E-mail: j.e.k.r.hentzen@umcg.nl

Copyright: (C) the author(s), publisher and licensee Medip Academy. This is an open-access article distributed under the terms of the Creative Commons Attribution Non-Commercial License, which permits unrestricted non-commercial use, distribution, and reproduction in any medium, provided the original work is properly cited.

\section{ABSTRACT}

Background: Surgery-related muscle loss (SRML) occurs in at least one out of three cancer patients within one week after major surgery. Though, this important phenomenon has hardly been investigated.

Methods: The MUSCLE POWER is a prospective, observational cohort study that investigates the presence, impact, and predictors for clinically relevant SRML in 178 cancer patients after major abdominal surgery using ultrasound measurements, squeeze and force measurements, and QoL questionnaires. Primary endpoint is the proportion of patients with clinically relevant SRML defined as $\geq 5 \%$ muscle loss within one week after surgery, measured by the cross-sectional area (CSA) of three different muscles: m. biceps brachii, m. rectus femoris, and m. vastus intermedius. Possible correlation with QoL and fatigue up to six months after surgery will be investigated. Daily physical activity during hospital stay will be monitored by a motility tracker, and protein intake will be monitored by a dietician. Possible predictors for clinically relevant SRML - consisting of age $\geq 65$ years, preoperative diabetes, preoperative sarcopenia, major postoperative complications (Clavien-Dindo $\geq$ III), insufficient physical activity, and insufficient postoperative protein intake - will be investigated with a multivariable logistic regression analyses with a backward stepwise approach. Variables with a $p<0.05$ will be retrained in the final multivariable model.

Discussion: The MUSCLE POWER investigates the presence and impact of clinically relevant SRML in cancer patients after major abdominal surgery. Crucial information regarding possible predictors for clinically relevant SRML can be used in future intervention studies to prevent postoperative muscle loss and subsequently improve postoperative outcome and QoL.

Trial Registration: Medical Ethics Committee of the University Medical Center Groningen, the Netherlands (METc2018/361, version 3.0, January 21, 2019), and Netherlands Trial Register ([NTR], NTR NL7505, version 1.0, February 7, 2019).

Keywords: Surgery-related muscle loss, Abdominal cancer surgery, Nutrition, Physical activity, Quality of life 


\section{INTRODUCTION}

Acute muscle loss has been studied extensively in critically ill patients in the intensive care unit (ICU) and is recognized as a common problem. ${ }^{1-7}$ At least $25 \%$ of the patients will develop ICU-acquired paresis, which is associated with significant mortality and morbidity and predicts long-term functional disability. ${ }^{5-9}$

In contrast to the growing amount of knowledge about the impact of muscle loss in ICU patients, there have been only a few studies reporting the amount of muscle loss in patients after major surgery. ${ }^{10-13}$ In patients who underwent elective high-risk cardiothoracic surgery, 55\% developed quadriceps atrophy seven days after surgery. ${ }^{10}$ In another study, one out of three cancer patients developed clinically relevant surgery-related muscle loss (SRML) within one week after curative gastric cancer surgery. ${ }^{11}$ Clinically relevant SRML was associated with postoperative complications and a longer length of hospital stay. These findings were also confirmed in a study including 254 patients who underwent major hepatectomies with extrahepatic bile duct resections. ${ }^{12}$ In this study, patients with clinically relevant SRML within one week after surgery had a significantly higher rate of major postoperative complications and an increased surgery-related mortality risk. Quality of Life (QoL) and fatigue were decreased up to three months after surgery. Postoperative fatigue is one of the main complaints after surgery and its presence prevents patients from returning to work. Only one study investigated and identified two independent predictors for clinically relevant SRML (age $\geq 65$ years and preoperative diabetes). ${ }^{11}$ These few studies demonstrate that clinically relevant SRML might be a major problem for our current healthcare system based on its impact on several short-term postoperative problems and its postoperative impact on QoL and fatigue.

Prevention of clinically relevant SRML can be a promising strategy to improve morbidity and mortality and increase QoL after major surgery. Unfortunately, these days, there is still a lack of scientific knowledge regarding this topic. Therefore, we made the design of the MUSCLE POWER study to further investigate the presence, impact, and possible predictors for clinically relevant SRML in cancer patients who underwent major abdominal cancer surgery. With this obtained knowledge, future intervention studies can focus on the prevention of postoperative muscle loss and minimize its impact on different postoperative outcomes and QoL in the long term.

\section{METHODS}

\section{Study design}

The MUSCLE POWER study is an observational singlecentre prospective cohort study in an academic setting that evaluates the proportion of cancer patients with clinically relevant surgery-related muscle loss (SRML) after major abdominal cancer surgery by using bedside ultrasound measurements of the arms and legs. Clinically relevant SRML is defined as $\geq 5 \%$ muscle loss within one week after surgery measured by the cross-sectional area (CSA) of the different muscles. In addition, we explore the effects of clinically relevant SRML on different life domains of QoL and fatigue after surgery. Furthermore, we investigate six possible predictors for clinically relevant SRML, identified by current literature or expert opinion, to provide essential information for future intervention studies to prevent clinically relevant SRML and reduce the possible associated impact on short- and long-term outcomes after surgery. We hypothesize that $50 \%$ of our patient population will have clinically relevant SRML within one week after major abdominal cancer surgery. Predictors for developing clinically relevant SRML will be preoperative sarcopenia, preoperative diabetes, age $\geq 65$ years, occurrence of major postoperative complications, insufficient physical activity, and insufficient protein intake during the first week after surgery. Clinically relevant SRML will also be associated with fatigue and a reduced QoL three and six months after surgery.

This trial will run in the University Medical Center Groningen (UMCG) from April 2019 until the target sample size of 178 patients has been reached (probably at the end of 2020). Other hospitals might be invited to collaborate, depending on the recruitment rate during the first year of patient inclusion.

\section{Patient selection}

Adult patients scheduled for major abdominal cancer surgery based on an underlying malignancy of the liver, pancreas, bile duct, colon, rectum, or pseudomyxoma peritonei are eligible for this study. Potential eligible patients will be identified at the weekly multidisciplinary oncology meeting and screened and informed about the study by their surgeon in the outpatient clinic during standard preoperative visits. Patients who meet the inclusion and exclusion criteria and are interested to participate in the study will receive a patient information letter describing the aims, content, duration, and objections of the study as well as the risks of participating. The investigator will contact these patients within one week by telephone to provide further information and answer remaining questions. After this conversation, patients have two weeks to decide whether they would like to participate. For patients who want to participate, an informed consent form has to be signed prior to the surgical procedure. During the study period, patients can leave the study at any time for any reason without any consequences. The investigator can decide to withdraw a patient from the study for urgent medical reasons.

\section{Inclusion criteria}

To be eligible to participate in this study, a patient must meet all of the following inclusion criteria: 
- $\quad$ Age $\geq 18$ years;

- Able to read and understand the Dutch language;

- Diagnosed with or suspicion of a liver tumour (primary cancer or colorectal liver metastases), pancreatic malignancy, bile duct malignancy, colon tumour, rectum tumour, or pseudomyxoma peritonei;

- $\quad$ Scheduled for open major abdominal cancer surgery at UMCG, consisting of the following surgical procedures:

- cytoreductive surgery combined with hyperthermic intraperitoneal intraoperative chemotherapy (CRS with HIPEC);

- (sub) total pelvic exenteration;

- (sub) total colon resection;

- pylorus preserving pancreaticoduodenectomy (PPPD);

- whipple procedure (classic pancreaticoduodenectomy);

- (sub) total pancreatectomy;

- major liver resection defined as $\geq 3$ liver segments.

- Undergone a preoperative computed tomography (CT) of the abdomen to determine preoperative sarcopenia;

- Given informed consent to participate in the study.

\section{Exclusion criteria}

A potential eligible patient who meets any of the following exclusion cannot participate in this study: scheduled to undergo emergency resection; scheduled to undergo laparoscopic surgery; scheduled to undergo robotic surgery; and unable to co-operate and give informed consent.

\section{Study endpoints}

The primary endpoint of this study is the proportion of patients who have clinically relevant SRML, defined as $\geq 5 \%$ muscle loss within one week after surgery measured by the CSA of the different muscles with bedside ultrasound measurements. The secondary endpoints are related to the amount of loss of muscle mass per day after surgery and during hospital stay and to the amount of decrease in muscle strength per day after surgery and during hospital stay. Six possible predictors for clinically relevant SRML-consisting of age $\geq 65$ years, preoperative diabetes, preoperative sarcopenia, major postoperative complications (Clavien-Dindo $\geq$ III), insufficient physical activity, and postoperative protein intake - will be explored. In addition, we will evaluated the amount of unplanned readmissions within 30 days after discharge, and the QoL and fatigue three and six months after surgery between patients with and without clinically relevant SRML.

\section{Sample size calculation}

The sample size calculation is based on the primary endpoint, the proportion of patients with clinically relevant SRML defined as $\geq 5 \%$ muscle loss within one week after surgery measured by the CSA of the different muscles. To determine the appropriate sample size (SS) for estimating the proportion of patients with clinically relevant SRML, we used the following formula:

$\mathrm{SS}=(\mathrm{Z} \text {-score })^{2} \times$ proportion $\times(1-$ proportion $) /($ margin of error $)^{2}$

For a confidence level of $95 \%, \alpha$ is 0.05 and the corresponding $\mathrm{Z}$-value is 1.96 . The sample proportion is unknown. We chose the number 0.50 (50\%) because it takes the maximum spread into account. Consensus about the margin of error was achieved by joint discussion of the research group; a margin error of 0.075 (7.5\%) was accepted. In total, 178 patients will be enrolled in the study to reach the target sample size.

\section{Measurements}

Figure 1 shows an overview of the different types of measurements during the study period.

\section{Muscle mass}

Baseline muscle mass for each patient will be measured by the investigator one day prior to surgery with a handheld ultrasound system (Philips FUS6882 Lumify L12-4) and consist of ultrasound measurements of the following four muscles: $\mathrm{m}$. biceps brachii, $\mathrm{m}$. rectus abdominis, $\mathrm{m}$. rectus femoris, and $\mathrm{m}$. vastus intermedius. For each muscle, the cross-section (anterior-posterior diameter) and the CSA will be measured bilaterally three times. Patients will be positioned supine on the bed with arm and leg muscles relaxed. The transducer will be placed perpendicular to the long axis of the different muscles (i.e. perpendicular to the major axis of the limb). The location of the measurement of the arm will be at twothirds of the length between the tip of the acromion and elbow fold with the elbow extended and the forearm in supine position. In the leg, the measurement will be at one-half the distance between spina iliaca anterior superior and the proximal border of the patella. The rectus will be measured halfway between the xiphoid and umbilicus on both sides of the abdomen. These different measuring points will be marked with a waterproof marker to ensure fixed points during the rest of the study period.

The muscle ultrasound measurements will be repeated on the third, seventh, and tenth day after surgery and on the day of discharge, with exception of the $\mathrm{m}$. rectus abdominis, because of the laparotomy wound that will occur after surgery. Baseline measurements and measurements obtained on the seventh day after surgery will be used to investigate the primary endpoint. Other measurements will be used the amount of loss of muscle mass per day after surgery and during hospital stay. 


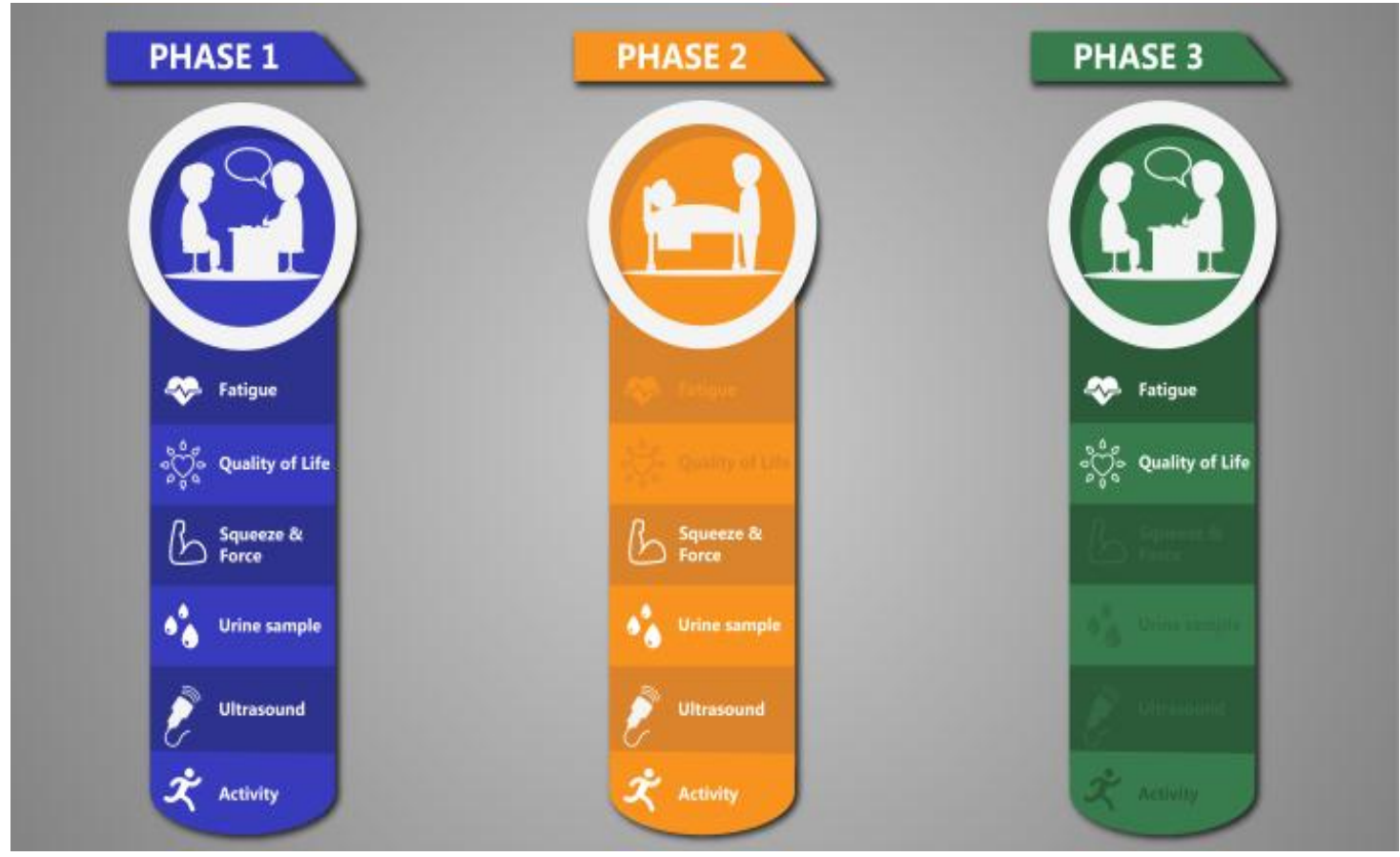

Figure 1: Study design. Phase 1: one day prior to surgery, phase 2: weeks after surgery till discharge, and phase 3: three and six months after surgery.

Table 1: Body position of the hand-held dynamometer (HHD) and the patient during different break tests.

\begin{tabular}{|c|c|c|c|}
\hline & Joint/limb position & Localization HHD & Position patient \\
\hline Elbow flexion & $\begin{array}{l}\text { Neutral shoulder, elbow flexed } 90^{\circ} \text {, } \\
\text { upper arm against trunk }\end{array}$ & $\begin{array}{l}\text { Just proximal to styloid process of } \\
\text { radius }\end{array}$ & Lying supine \\
\hline Elbow extension & Same as in flexion & Just proximal to ulnar head & Same as in flexion \\
\hline Knee flexion & Hip and knee flexed $90^{\circ}$ & Just proximal to calcaneus & Sitting in a chair \\
\hline Knee extension & Same as in flexion & Just proximal to talis & Same as in flexion \\
\hline
\end{tabular}

\section{Muscle strength}

Squeeze and force measurements will be performed on the same days as the ultrasound measurements: one day prior to surgery; third, seventh, and tenth day after surgery; and on the day of discharge.

Isometric muscle force from grip strength of the hand, elbow flexion and extension, and knee flexion and extension will be measured with a hand-held dynamometer (HHD) using different break tests. A description of the body positions of the HHD and the patient during different measurements is described in Table 1 and shown in Figure 2 (A and B) and Figure 3 (A and $\mathrm{B}$ ). Patients will receive the instruction to build up maximal strength in one to two seconds. The researcher will gradually overcome the muscle force and stop at the moment the extremity gives away. Each measurement will be carried out three times in series with 20 seconds intervals between the contractions. This is a standardized method for performing a break test. ${ }^{14}$

By using multiple squeeze and force measurements at different time points, we can analyse the amount of decrease in muscle strength per day, discover specific patterns of decrease in muscle strength, and calculate the total amount of decrease in muscle strength during the hospital stay.

\section{Physical activity}

Insufficient physical activity post operation might be a possible predictor for clinically relevant SRML. Therefore, each patient will wear a motility tracker (Actigraph wGT3X-BT [Actigraph, Pensacola, FL, USA]) during the first week after surgery except on the day of operation and during water-based activities. The Actigraph wGT3X-BT is a small and lightweight device that will be worn on the left or right ankle with an elastic belt to prevent discomfort for the patient and to measure physical activity data as reliably as possible. The device does not provide direct feedback to the patient.

The Actigraph provides high raw acceleration data to capture physical activity intensity, activity bouts, and sedentary bouts. The information from the activity tracker will be downloaded to ActiLife. This program automatically provides total activity counts and time per 
intensity level per day. Existing cut-off points for moderate (2020-5999 counts/min) and vigorous intensity physical activity (>5999 counts/min) will be used. A valid monitoring day will be defined as having 10 or more hours of monitor wear. Wear time is determined by subtracting non-wear time from 24 hours. Non-wear time is as defined by an interval of at least 60 consecutive min of zero activity intensity counts.

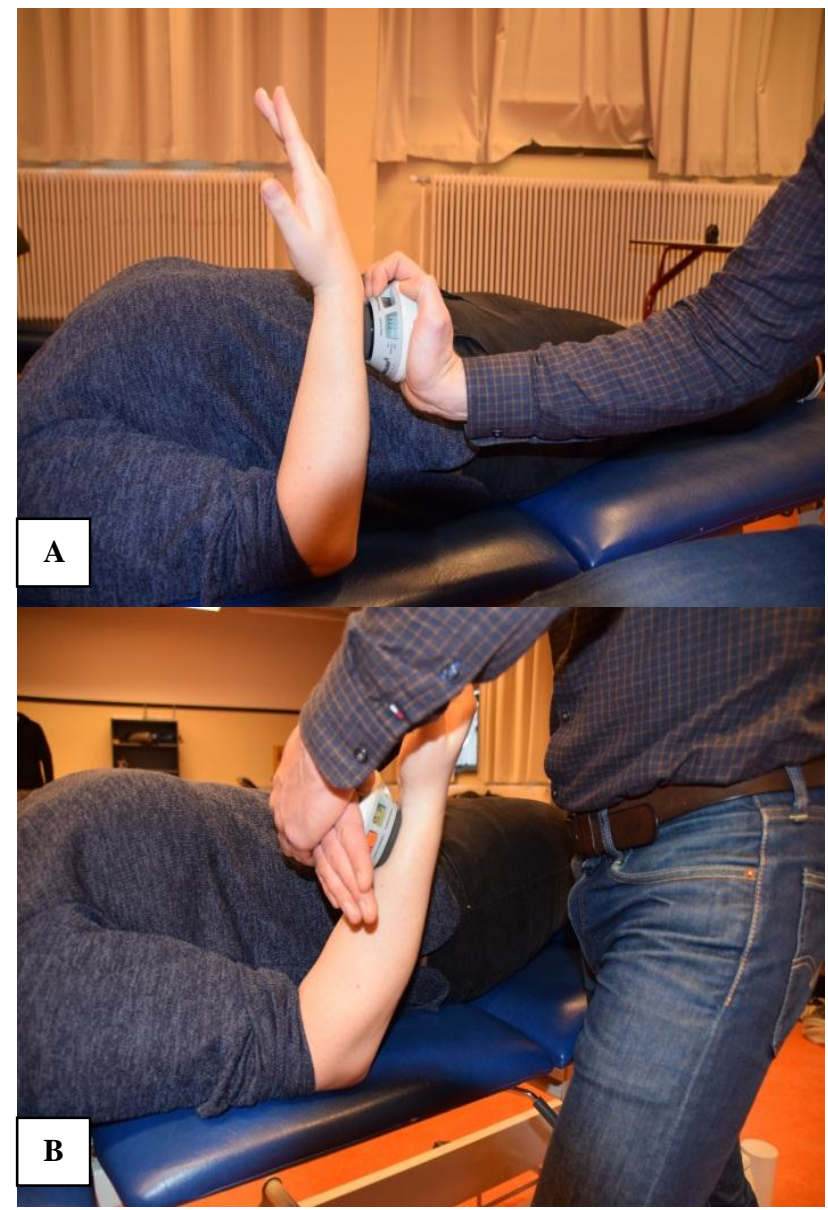

Figure 2: Positions of the hand held dynamometer for elbow flexion and extension. (A) Elbow flexion, (B) elbow extension.

The total amount of moderate and vigorous intensity physical activity in the first seven days after surgery will be calculated. If a patient's activity is less than $150 \mathrm{~min}$ of moderate/vigorous intensity physical activity within the first week after surgery, it will be registered as 'insufficient physical activity'. We will than investigate if insufficient physical activity is associated with clinically relevant SRML.

\section{Protein intake}

We suspect that malnutrition prior to surgery and insufficient protein intake during the first week after surgery might be possible predictors for clinically relevant SRML. According to the European Society for Clinical Nutrition and Metabolism guidelines, surgical patients need $1.5 \mathrm{~g} / \mathrm{kg}$ protein per day after major surgery. ${ }^{15}$ Patients will fill in the patient-generated subjective global assessment (PG-SGA) questionnaire one day prior to surgery. This validated questionnaire for nutritional assessment focuses on four elements: (1) dietary intake change, (2) gastrointestinal symptoms, (3) short-term weight loss, and (4) changes in functional capacity. ${ }^{16}$ Each element can be scored from zero to four. The higher the total score from the PG-SGA, the greater the risk for malnutrition, in which a score of nine or higher indicates a critical need for nutritional interventions.

The first seven days after surgery, daily protein and energy intake will measured by a dietician by using a nutrition dairy. The intake on the day of the surgical procedure itself will be excluded. Afterwards, we will calculate the number of days that the protein intake was not adequate (e.g., $<1.5 \mathrm{~g} / \mathrm{kg}$ ). We will register the protein intake as 'insufficient' if the patient received less than $1.5 \mathrm{~g} / \mathrm{kg}$ for two or more days within the first week after surgery. Next we investigate if an insufficient amount of protein intake during the first week after surgery is associated with clinically relevant SRML.

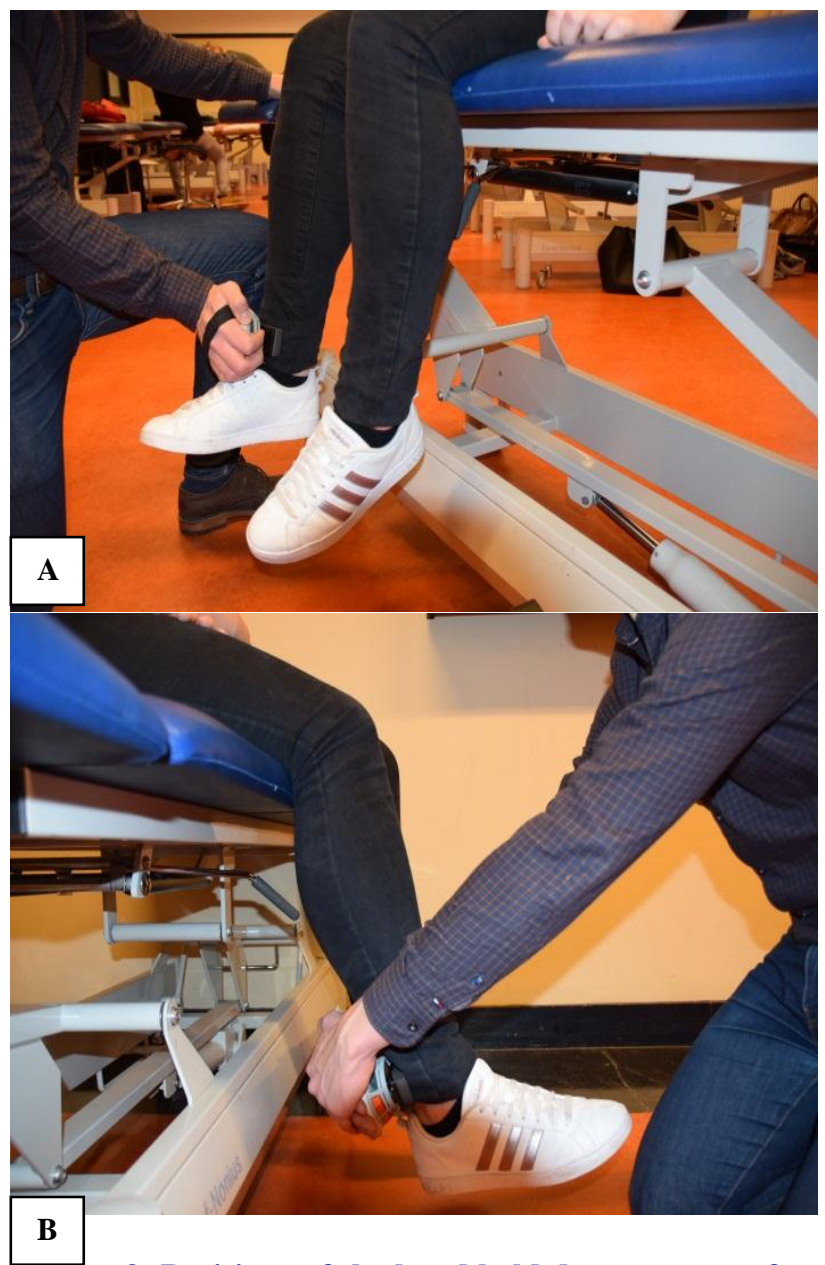

Figure 3: Positions of the hand held dynamometer for knee flexion and extension. (A) Knee flexion, (B) knee extension. 


\section{Sarcopenia preoperatively}

We suspect that preoperative sarcopenia might be a possible predictor for clinically relevant SRML. The presence of preoperative sarcopenia will be measured on preoperative workup CT scans by an experienced musculoskeletal radiologist. Cross-sectional skeletal muscle surface $\left(\mathrm{cm}^{2}\right)$ will be assessed at the level of the third lumbar vertebra (L3) to determine the sarcopenia index. Measurements of the psoas and abdominal wall will be obtained and compared. Skeletal muscle cut-off values for sarcopenia will be corrected for height, age, and ethnic group according to the consensus diagnostic criteria for sarcopenia, developed by the European Working Group on Sarcopenia in Older People (EWGSOP). ${ }^{17}$ These measurements will be used to investigate if preoperative sarcopenia is associated with clinically relevant SRML.

\section{Other possible predictors}

In addition to the presence of insufficient physical activity, insufficient protein intake, and preoperative sarcopenia, we identified three other possible predictors for clinically relevant SRML: age $\geq 65$ years, preoperative diabetes, and occurrence of major postoperative complications. Data about these possible predictors will be collected from digital patient records. Postoperative complications will be registered using the Clavien-Dindo classification system. ${ }^{18}$ Major postoperative complications are defined as Clavien-Dindo $\geq$ III. All possible predictors will be dichotomized (presence or absence of the predictor) for further analyses.

\section{Urine collection}

Urinary creatinine excretion rate (CER) measured from a 24-hour sample is an established non-invasive marker of total body muscle mass and seems to predict long-term outcomes in patients after liver transplantation. ${ }^{19} \mathrm{We}$ suspect that CER might be an interesting marker in predicting clinically relevant SRML. Therefore, collection of 24-hour urine samples will take place at baseline and be repeated on the seventh day after surgery.

\section{Questionnaires}

Patients will receive three different questionnaires at baseline and three and six months after surgery to compare scores over time to identify the impact of clinically relevant SRML on QoL and fatigue. Patients will receive the questionnaires by e-mail or on paper.

The WHOQOL-Bref (version 1.0, December 1996) and RAND-36 (version 2.0, 2007) will be used to assess QoL. The WHOQOL-Bref contains a total of 26 questions including the following four domains: physical health, psychological, social relationships, and environment. ${ }^{20,21}$ The RAND-36 Item Health Survey cover eight concepts: physical functioning, bodily pain, role limitations due to physical health problems, role limitations due to personal or emotional problems, role emotional well-being, social functioning, energy/fatigue, and general health perceptions. ${ }^{22}$ It also includes a single item that provides an indication of perceived change in health. Both questionnaires are available in Dutch.

The multidimensional fatigue inventory (Dutch version MFI-20, 2003) will be used to assess fatigue. The MFI-20 is a 20-item self-report instrument designed to measure fatigue and is well-established in cancer patients. ${ }^{23}$ It covers the following dimensions: general fatigue, physical fatigue, reduced activity, reduced motivation, and mental fatigue.

\section{Data handling}

All data will be handled confidentially and stored in an electronic case record form designed in the software program Open Clinica (TRAIT BV, the Netherlands), a program especially designed for clinical trial data recording and monitoring. Only the principal investigators will have access to the stored data.

\section{Data analysis plan}

All statistical analyses will be conducted using SPSS Statistics version 24.0 (IBM Corporation, Armonk, NY, USA). Continuous variables will be expressed as mean \pm standard deviation (SD) or median with interquartile range (IQR) and categorical variables will be described as count $(n)$ and percentage (\%) with $95 \%$ confidence intervals (CI). Patient characteristics will be compared using the student $t$-test or Mann Whitney U-test for continuous variables and differences between nominal variables will be determined using Pearson chi-square test or Fisher's exact test. Distribution will be assessed with the Shapiro-Wilk normality test.

The different possible predictors for clinically relevant SRML will be investigated with a multivariable logistic regression analyses to calculate odds ratios (ORs) with 95\% Cl. A backwards stepwise selection methodology will be used to identify independent predictors for clinically relevant SRML. Variables with $p<0.1$ in the univariate analysis will be included in the multivariate analysis, and variables significant at $p<0.05$ will be retrained in the final multivariable model. All tests will be two-sided and $p \leq 0.05$ will be considered statistically significant.

\section{Dissemination policy}

Both positive and negative research results will be disclosed and submitted to peer-reviewed scientific journals. The principal investigator and steering committee will prepare the manuscripts together with the statistician and other active writing committee members. Co-authorship is reserved for all investigators and in addition to those who constructively contributed to the 
study at the discretion of the project leader and steering committee. Finally, disputes on the interpretation of the results may not lead to an unnecessary delay in publication.

\section{DISCUSSION}

The results from the MUSCLE POWER study will provide important clinical knowledge on the presence, impact, and possible predictors for clinically relevant SRML after major abdominal cancer surgery. This obtained scientific knowledge, will fill in important gaps in the current literature and may give leads how to prevent clinically relevant SRML and improve morbidity and mortality and QoL after surgery in this vulnerable patient population.

The primary aim of this study is to identify the proportion of patients who have clinically relevant SRML, defined in this study as $\geq 5 \%$ muscle loss within one week after surgery measured by the CSA of three different muscles by ultrasound. Our primary endpoint is clear and relatively easy to measure, although there is currently no global consensus about what amount of muscle loss is clinically relevant (e.g. harmful for the patient). The definition of clinically relevant SRML is therefore based on combining data from three previous published studies that examined acute muscle loss in surgical patients. ${ }^{10-12}$

In most previous clinical studies, changes in skeletal muscle mass were investigated by $\mathrm{CT}$ scans at different time points. ${ }^{3,4,11,12}$ In the meantime, muscle ultrasound has emerged as a common, inexpensive, reliable, and valid imaging technique for measuring skeletal muscle at the bedside in patients with different clinical conditions, without exposing patients to harmful ionizing radiation. ${ }^{1,9,24-27}$ Therefore, in the present study, we will investigate changes in skeletal muscle mass by using bedside ultrasound measurements instead of routinely performing additional CT scans at different time points.

In the MUSCLE POWER study, additional important information about two factors that may extensively influence skeletal muscle loss will be collected as well. To maintain skeletal muscle tissue, food intake and muscle contraction are crucial. ${ }^{28}$ Previous published papers show that in the majority of patients after surgery, daily protein intake is much lower than the recommended guidelines of $1.5 \mathrm{~g} / \mathrm{kg} / \mathrm{day} .{ }^{13,17,18,29}$ In the present study, protein intake will be monitored daily by a dietician during the first week after surgery to explore its impact on clinically relevant SRML. Additionally, all patients will wear a motility tracker to calculate daily moderate and vigorous physical activity, as hospitalization is known to be associated with reduced levels of physical activity. Recent studies with older patients show that nutritional and physical interventions should be combined to minimize loss of muscle mass and muscle strength. ${ }^{30-32}$ To the best of our knowledge, continuous monitoring of both factors in patients in the first week after abdominal cancer surgery has not yet been performed. We suspect that in the near future, data from the MUSCLE POWER study may play an important role in the development of new nutritional and physical strategies to prevent postoperative muscle loss.

Of course, our study protocol may bear some limitations. First of all, the sample size calculation was based on the scarcely available scientific data about clinically relevant SRML. ${ }^{10-12}$ Our hypothesis that $50 \%$ of our patient population will have clinically relevant SRML after major abdominal cancer surgery will take the limited amount of previous available data and the maximum spread into account. Despite this, the present study may not be powered enough to investigate all six possible predictors for clinically relevant SRML that were identified by current literature or expert opinion. Another limitation is the fact that we do not perform echointensity assessments of the different muscles to investigate muscle quality. Specific trainings are necessary to reliably reproduce these measurements and software that is necessary for echogenicity analysis is not available on our ultrasound system. ${ }^{33,34}$ To partially solve this problem, we will perform squeeze and force measurements to obtain some information about muscle function (e.g., muscle strength).

Funding: This work is supported by a grant from the UMCG Cancer Research Foundation (Institutional Foundation for Cancer Research and Development). The study protocol has not undergone any peer-review by this funding body, nor will they play a role in the analysis and interpretation of the data

Conflict of interest: None declared

Ethical approval: The study protocol has been approved by the Medical Ethics Committee of the University Medical Center Groningen, the Netherlands (METc2018/361, version 3.0, January 21, 2019), and the study protocol was registered with the Netherlands Trial Register ([NTR], NTR NL7505, version 1.0, February 7, 2019)

\section{REFERENCES}

1. Puthucheary ZA, Rawal J, McPhail M, Connolly B, Ratnayaka G, Chan P, et al. Acute skeletal muscle wasting in critical illness. JAMA 2013;310:1591600.

2. Campbell IT, Watt T, Withers D, England R, Sukumar S, Keegan MA, et al. Muscle thickness, measured with ultrasound, may be an indicator of lean tissue wasting in multiple organ failure in presence of edema. Am J Clin Nutr. 1995;62:533-9.

3. Weijs PJ, Looijaard WG, Dekker IM, Stapel SN, Girbes AR, Oudemans-van Straaten HM, et al. Low skeletal muscle area is a risk factor for mortality in mechanically ventilated critically ill patients. Crit Care. 2014;18:R12.

4. Moisey LL, Mourtzakis M, Cotton BA, Premji T, Heyland DK, Wade CE, et al. Skeletal muscle 
predicts ventilator-free days, ICU-free days, and mortality in elderly ICU patients. Crit Care. 2013; 17:R206.

5. de Jonghe B, Sharshar T, Lefaucheur JP, Authier FJ, Durand-Zaleski I, Boussarsar $\mathrm{M}$, et al. Paresis acquired in the intensive care unit: a prospective multicenter study. JAMA 2002;288:2859-67.

6. Chambers MA, Moylan JS, Reid MB. Physical inactivity and muscle weakness in the critically ill. Crit Care Med. 2009;37:337-46.

7. Latronico N, Bolton CF. Critical illness polyneuropathy and myopathy: a major cause of muscle weakness and paralysis. Lancet Neurol. 2011;10:931-41.

8. Baldwin CE, Bersten AD. Alterations in respiratory and limb muscle strength and size in patients with sepsis who are mechanically ventilated. Phys ther. 2014;94:68-82.

9. Dos Santos C, Hussain SN, Mathur S, Picard M, Herridge M, Correa J, et al. Mechanism of chronic muscle wasting and dysfunction after an intensive care unit stay: a pilot study. Am J Respir Crit Care Med. 2016;194:821-30.

10. Bloch SA, Lee JY, Wort SJ, Polkey MI, Kemp PR, Griffiths MJ. Sustained elevation of circulating growth and differentiation factor-15 and a dynamic imbalance in mediators of muscle homeostasis are associated with the development of acute muscle wasting following cardiac surgery. Crit Care Med. 2013;41:982-9.

11. Huang DD, Ji YB, Zhou DL, Li B, Wang SL, Chen $\mathrm{XL}$, et al. Effect of surgery-induced acute muscle wasting on postoperative outcomes and quality of life. J Surg Res. 2017;218:58-66.

12. Otsuji H, Yokoyama Y, Ebata T, Igami T, Sugawara G, Mizuno T, et al. Surgery-related muscle loss and its association with postoperative complications after major hepatectomy with extrahepatic bile duct resection. World J Surg. 2017;41:498-507.

13. Kouw IWK, Groen BBL, Smeets JSJ, Kramer IF, van Kranenburg JMX, Nilwik R, et al. One week of hospitalization following elective hip surgery induces substantial muscle atrophy in older patients. JAMDA. 2019;20:35-42.

14. Douma RKW, Soer R, Krijnen WP, Reneman M, van der Schans CP. Reference values for isometric muscle force among workers for the Netherlands: a comparison of reference values. BMC Sports Science Medicine and Rehabilitation. 2014;6:10.

15. Singer P, Reintam Blaser A, Berger MM, Alhazzani W, Calder PC, Casaer MP, et al. ESPEN guideline on clinical nutrition in the intensive care unit. Clinical Nutrition. 2018.

16. Ottery D. Patient Generated Subjective Global Assessment. In: McCallum P, Polisena C, Ed. The clinical guide to oncology nutrition, The American Dietetic Association, Chicago; 11-23.

17. Cruz-Jentoft AJ, Baeyens JP, Bauer JM, Boirie Y, Cederholm T, Landi F, et al. Sarcopenia: European consensus on definition and diagnosis: report of the
European Working Group on Sarcopenia in Older People. Age and Ageing. 2010;39:412-23.

18. Clavien PA, Barkun J, de Oliveira ML, Vauthey JN, Dindo D, Schullick RD, et al. The Clavien-Dindo classification of surgical complications: five-year experience. Ann Surg. 2009;250:187-96.

19. Stam SP, Osté MCJ, Eisenga MF, Blokzijl H, van den Berg AP, Bakker SJL, et al. Posttransplant muscle mass measured by urinary creatinine excretion rate predicts long-term outcomes after liver transplantation. Am J Transplant. 2018;19:54050.

20. The WHOQOL group. Development of the World Health Organization WHOQOL-BREF quality of life assessment. Psychol Med. 1998;28:551-8.

21. Skevington SM, Lotfy M, O'Connell KA, WHOQOL group. The World Health Organization's WHOQOL-BREF quality of life assessment: psychometric properties and results of the international field trial. A report from the WHOQOL group. Qual Life Res. 2004;13:299-310.

22. Van der Zee K, Sanderman R, Heyink JW, de Haes H. Psychometric qualities of the RAND 36-item Health Survey 1.0: a multidimensional measure of general health status. Int J Behav Med. 1996;3:10422.

23. Smets EM, Garssen B, Bonke B, De Haes JC. The Multidimensional Fatigue Inventory (MFI) psychometric qualities of an instrument to assess fatigue. J Psychosom Res. 1995;39:315-25.

24. Bunnell A, Ney J, Gellhorn A, Hough CL. Quantitative neuromuscular ultrasound in intensive care unit-acquired weakness: a systematic review. Muscle Nerve 2015;52:701-8.

25. Connolly B, MacBean V, Crowley C, Lunt A, Moxham J, Rafferty GF, et al. Ultrasound for the assessment of peripheral skeletal muscle architecture in critical illness: a systematic review. Crit Care Med. 2014;7:1-10.

26. Sabatino A, Regolisti G, Bozzoli I, Fani F, Antoniotti R, Maggiore U, et al. Reliability of bedside ultrasound for measurement of quadriceps muscle thickness in critically ill patients with acute kidney injury. Clin Nutr. 2017;36:1710-5.

27. Parry SM, El-Ansary D, Cartwright MS, Sarwal A, Berney S, Koopman R, et al. Ultrasonography in the intensive care setting can be used to detect changes in the quality and quantity of muscle and is related to muscle strength and function. J Crit Care. 2015;30:1151.

28. Koopman R, van Loon LJ. Aging, exercise, and muscle protein metabolism. J Appl Physiol. 2009;106:2040-8.

29. Gillis C, Nguyen TH, Liberman AS, Carli F. Nutrition adequacy in enhanced recovery after surgery: a single academic center experience. Nutr Clin Pract. 2015;30:414-9.

30. English KL, Paddon-Jones D. Protecting muscle mass and function in older adults during bed rest. Curr Opin Clin Nutr Metab Care 2010;13:34-39. 
31. Paddon-Jones D, Sheffield-Moore M, Urban RJ, Sanford AP, Aarsland A, Wolfe RR, et al. Essential amino acid and carbohydrate supplementation ameliorates muscle protein loss in humans during 28 days bedrest. J Clin Endocrinol Metab. 2004;89:4351-8.

32. Wall BT, van Loon LJC. Nutritional strategies to attenuate muscle disease atrophy. Nutr Rev. 2013;71:195-208.

33. Ticinesi A, Meschi T, Narici MV, Laurentani F, Maggio M. Muscle ultrasound and sarcopenia in older individuals: a clinical perspective. J Am Med Dir Assoc. 2017; 18:290-300.
34. Berger J, Bunout D, Barrera G, de la Maza MP, Henriquez S, Leiva L, et al. Rectus femoris (RF) ultrasound for the assessment of muscle mass in older people. Arch. Gerontol. Geriatr. 2015;61:33-8.

Cite this article as: Hentzen JEKR, van Wijk L, Buis CI, Viddeleer AR, de Bock GH, van der Schans CP, et al. Impact and risk factors for clinically relevant surgeryrelated muscle loss in patients after major abdominal cancer surgery: study protocol for a prospective observational cohort study (MUSCLE POWER). Int J Clin Trials 2019;6(3): 138-46. 